\title{
Comparison of mitochondrial A3243G mutation loads in easily accessible samples from a family with maternally inherited diabetes and deafness
}

\author{
TOSHIYUKI FUKAO ${ }^{1,2}$, MASASHI KONDO ${ }^{1}$, TAKAHIRO YAMAMOTO ${ }^{1}$, \\ KENJI E. ORII ${ }^{1}$ and NAOMI KONDO ${ }^{1}$ \\ ${ }^{1}$ Department of Pediatrics, Graduate School of Medicine, Gifu University; \\ ${ }^{2}$ Medical Information Sciences Division, United Graduate School of Drug Discovery and \\ Medical Information Sciences, Gifu University, Gifu 501-1194, Japan
}

Received August 19, 2008; Accepted October 3, 2008

DOI: $10.3892 / \mathrm{mmr} 00000063$

\begin{abstract}
The mitochondrial A3243G mutation is most commonly related to the MELAS syndrome, but can cause many different clinical manifestations at various ages. Here, we present a family with maternally inherited diabetes and deafness (MIDD), the proband of which exhibits hearing loss, diabetes mellitus, cardiomyopathy and short stature. Four easily accessible samples (whole blood, hair roots, buccal scrapings and urinary sediment) from the proband and her 3 sons were simultaneously analyzed for heteroplasmic percentages of the A3243G mutation in their DNA. More than 10 subclones were sequenced and the percentage of clones possessing the A3243G mutation was calculated. The proportion of mutant genomes varied widely among the four samples tested. Blood DNA consistently had the lowest percentage of mutation load, while urinary sediment tended to have the highest. The 3 sons have not exhibited hearing disability or diabetes mellitus thus far. However, their mutation loads in all 4 tissue samples were higher than those in the corresponding samples from the proband. Follow-up of this family over time is necessary to understand the relationship between the heteroplasmic mutation loads in the 4 different samples and the clinical manifestations of MIDD/MELAS.
\end{abstract}

\section{Introduction}

Goto et al identified the A3243G mutation in the tRNALeu (UUR) gene of mitochondrial DNA in patients with MELAS (1). This same mutation can cause other clinical manifestations

Correspondence to: Professor Toshiyuki Fukao, Department of Pediatrics, Graduate School of Medicine, Gifu University, Yanagido1-1, Gifu 501-1194, Japan

E-mail: toshi-gif@umin.net

Key words: A3243G, MELAS, maternally inherited diabetes and deafness, mutation load, heteroplasmy of mitochondrial multisystem disorders, such as maternally inherited diabetes and deafness (MIDD), myoclonus epilepsy with ragged red fibers, progressive external ophthalmoplegia, hypertrophic cardiomyopathy and the Leigh syndrome (2). The A3243G mutation load in different organs and tissues in association with heteroplasmy may explain which clinical presentations occur among patients who carry the mutation (2-5). Usually, DNA from blood leukocytes or muscle biopsy samples is subjected to mitochondrial mutation analysis. Although the heteroplasmic percentages of mutant mitochondrial DNA vary among tissues, it is difficult to speculate on the heteroplasmic percentages of mutant mitochondrial DNA in a specific organ (such as skeletal muscle, the brain and the pancreatic islet). Results from leukocyte samples can supply information on the heteroplasmic mutation loads in leukocytes, which is clinically useful to some extent $(4,6,7)$. However, the usefulness of DNA from other easily accessible samples (hair roots, buccal scraping and urinary sediment) has been insufficiently evaluated.

In this study, we performed A3243G mutation analysis using DNA samples from the whole blood, urinary sediment, buccal scraping and hair roots of a MIDD family, the proband of which exhibits diabetes mellitus, hearing loss, cardiomyopathy and short stature.

\section{Patients and methods}

Patients. The proband was affected with diabetes mellitus from the age of 24 and with hearing loss from the age of 28 years. She has a height of $145 \mathrm{~cm}$ and weight of $40 \mathrm{~kg}$. Her mother, maternal grandmother and maternal cousin also had diabetes mellitus and hearing loss. The proband additionally developed hypertrophic cardiomyopathy, evident from the age of 33 years. She was diagnosed as having MIDD caused by the A3243G mutation. She has three sons, aged 13 (Son 1), 9 (Son 2) and 8 (Son 3) years. After the two elder boys were revealed to have the WPW syndrome and cardiomyopathy, the family was referred to our hospital for further evaluation. Their clinical presentations are summarized in Table I. Informed consent to analyze their DNA for mitochondrial mutations was obtained from the mother and her sons. 
Table I. Case presentations.

\begin{tabular}{|c|c|c|c|c|}
\hline Case & Proband & Son 1 & Son 2 & Son 3 \\
\hline \multicolumn{5}{|l|}{ Central nervous system } \\
\hline Stroke-like episode & - & - & - & - \\
\hline Mental retardation & - & + & + & - \\
\hline Retinal degeneration & - & - & - & - \\
\hline Convulsion & - & - & - & - \\
\hline Hearing loss & + & - & - & - \\
\hline Myoclonus & - & - & - & - \\
\hline Migraine headache & + & - & - & + \\
\hline \multicolumn{5}{|l|}{ Skeletal muscle } \\
\hline External ophthalmoplegia & - & - & - & - \\
\hline Ptosis & - & - & - & - \\
\hline Myopathy & - & - & - & - \\
\hline \multicolumn{5}{|l|}{ Metabolism } \\
\hline Hyperlactic acidemia & + & + & + & + \\
\hline \multicolumn{5}{|l|}{ Other } \\
\hline Renal tubular dysfunction & - & - & - & - \\
\hline Anemia & - & - & - & - \\
\hline WPW syndrome & + & + & + & - \\
\hline Hypertrophic cardiomyopathy & + & + & + & - \\
\hline Short stature & + & + & + & - \\
\hline Diabetes mellitus & + & - & - & - \\
\hline Hypoparathyroidism & - & - & - & - \\
\hline Liver dysfunction & - & - & - & - \\
\hline
\end{tabular}

Table II. Proportion (\%) of the A3243G mutation in the 4 samples.

\begin{tabular}{llrrr}
\hline Case & Proband & Son 1 & Son 2 & Son 3 \\
\hline Whole blood & $4 / 12(33)$ & $7 / 12(58)$ & $6 / 11(55)$ & $8 / 12(67)$ \\
Hair roots & $7 / 10(70)$ & $14 / 16(88)$ & $10 / 10(100)$ & $9 / 12(75)$ \\
Buccal scraping & $7 / 12(58)$ & $8 / 11(73)$ & $9 / 12(75)$ & $10 / 11(91)$ \\
Urinary sediment & $8 / 12(67)$ & $11 / 12(92)$ & $12 / 12(100)$ & $12 / 12(100)$ \\
\hline
\end{tabular}

Subclones $(>10)$ were sequenced to calculate the mutation load for each sample. Number of A3243G mutant subclones/number of total subclones (percentage of mutation load).

DNA extraction. DNA was extracted from $1 \mathrm{ml}$ of whole blood, from urinary sediment from $50 \mathrm{ml}$ of urine samples, from buccal scrapings using a cotton-tipped swab and from three hair roots using the Sepa Gene kit (Sanko Junyaku, Tokyo, Japan). Glycogen was added to isopropanol DNA precipitation to avoid the loss of DNA.

A3243G detection. PCR amplification of a 296-bp fragment including the $\mathrm{A} 3243 \mathrm{G}$ site was performed using a primer pair (5'-AGGACAAGAGAAATAAGGCC-3' and 5'-CACGTTG GGGCCTTTGCGTA-3'). The amplified fragments were subjected to Apa I digestion followed by $2 \%$ agarose gel electrophoresis. The A3423G mutant fragment was digested into 184- and 112-bp fragments by Apa I. PCR fragments were also subcloned into T vectors. Subclones $(>10)$ were sequenced in order to calculate the mutation load for each sample.

\section{Results}

The proband was diagnosed as having MIDD based on clinical phenotypes (Table I), family history and the presence of the mitochondrial A3243G mutation. Since her 3 sons also presented some clinical indicators and symptoms (Table I), their samples were analyzed along with those of the proband. 


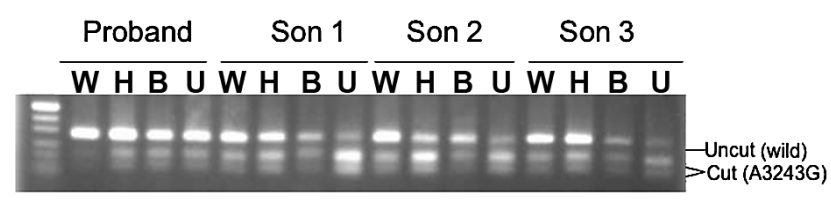

Figure 1. A3243G mutation loads in the 4 samples. W, whole blood; H, hair roots; $\mathrm{B}$, buccal scraping and $\mathrm{U}$, urinary sediment. A fragment encompassing the A3243G site was digested into 184- and 112-bp fragments by Apa I and electrophoresed on a $2.0 \%$ agarose gel. Normal fragments (without the A3243G mutation) could not be digested by Apa I.

A mitochondrial DNA fragment including the A3243G mutation was successfully amplified, not only from the whole blood samples but also from the hair roots, buccal scrapings and urinary sediment. A3243G mutant fragments were digested into two smaller fragments by Apa I. Normal fragments could not be digested by Apa I. The A3243G mutation loads differed depending on the tissue and individual involved (Fig. 1).

To calculate what percentage of mitochondrial DNA possessed the $\mathrm{A} 3243 \mathrm{G}$ mutation, we sequenced $>10$ subclones and calculated the percentage of those possessing the A3243G mutation. The proportion of mutant genomes varied widely among the 4 samples tested (Table II). The 3 sons have not exhibited hearing disability or diabetes mellitus thus far. However, their mutation loads in all 4 tissue samples were higher than those in the corresponding samples from the proband. Blood DNA consistently had the lowest percentage of mutation load, while urinary sediment tended to have the highest.

\section{Discussion}

One characteristic of a mitochondrial mutation is its great phenotypic diversity within a family and among families. This is due to variance in the heteroplasmic percentages of mutant mitochondrial DNA among different tissues, as seen in different family members. Complicating matters is the fact that the mutation load in a specific organ cannot be ascertained based on results obtained from other tissues (1).

To some extent, however, information on heteroplasmic mutation loads in leukocytes is clinically useful $(4,6,7)$. The heteroplasmic level of A3243G in blood cells was reported to be considerably correlated with age at onset of diabetes and deafness (6). Lu et al also reported that heteroplasmic A3243G mutation levels appeared to be correlated with the severity and age at onset of diabetes in a MIDD family (7). In the family presented here, the $\mathrm{A} 3243 \mathrm{G}$ the mutation load was much higher in the whole blood of the 3 sons (58-70\%), who have not exhibited hearing disability or diabetes mellitus thus far, than in the proband (33\%). However, most MIDD patients develop diabetes mellitus between the ages of 15 and 70 years, probably because oxidative stress on the pancreas from the mitochondrial defect takes years to result in a clinically relevant insulin secretory defect. Therefore, the sons may still be in a subclinical state. The age factor is also important when evaluating mutation loads. A decrease in the heteroplasmic level of the $\mathrm{A} 3243 \mathrm{G}$ mutation occurs over time in mitotic tissues, such as blood leukocytes $(8,9)$ and epithelial cells (uterus cervical cells) (10). Heteroplasmic levels in leukocytes decline in conjunction with aging by $\sim 0.7 \%$ per year (11). Thus, it may be difficult to compare the mutation loads of the proband (mother) to those of her sons using blood samples as the DNA source, and thereby difficult to discuss genetic anticipation.

As discussed above, the mutation load in leukocytes has been well characterized, while mutation loads in other easily accessible clinical samples, such as hair roots, buccal scrapings and urinary sediment, have been less so. We determined the mutation loads within one family from these samples as well as from the whole blood. Blood DNA consistently had the lowest percentage of mutation load, while urinary sediment tended to have the highest. The mutation load was higher in all 4 tissue samples from the 3 sons than in the corresponding samples from the proband. Age-related changes in mutation load in these tissues have yet to be reported, with the exception of the whole blood (leukocytes).

There is only one precedent for mutation analysis using these 4 clinically accessible samples simultaneously. Shanske et al performed screening of the A3243G mutation in 5 different samples (leukocytes, fibroblasts, hair roots, urinary sediment and cheek mucosa) from a large number of patients harboring the A3243G mutation (12). They found that, as a rule, DNA obtained from urinary sediment had the highest and blood the lowest proportion of mutant genomes; individual hair roots differed markedly from one another, while fibroblasts and cheek mucosa tended to have higher mutation loads than blood but lower than urinary sediment. They also found that in some individuals the mutation was undetectable in blood but clearly present in other tissues, and concluded that urinary sediment and cheek mucosa are the samples of choice for the diagnosis of mitochondrial DNA mutations. Although our findings were obtained from a study of a MIDD family, they are in agreement with those of the abovementioned study. Follow-up of this family over time may help establish the relationships that exist between tissue-specific heteroplasmic mutation loads and clinical manifestations of MIDD/MELAS.

\section{Acknowledgements}

We thank N. Sakaguchi for technical assistance.

\section{References}

1. Goto Y, Nonaka I and Horai S: A mutation in the tRNA(Leu) (UUR) gene associated with the MELAS subgroup of mitochondrial encephalomyopathies. Nature 348: 651-653, 1990.

2. Finsterer J: Genetic, pathogenetic and phenotypic implications of the mitochondrial A3243G tRNALeu(UUR) mutation. Acta Neurol Scand 116: 1-14, 2007.

3. Koga Y, Koga A, Iwanaga R, Akita Y, Tubone J, Matsuishi T, Takane N, Sato Y and Kato H: Single-fiber analysis of mitochondrial A3243G mutation in four different phenotypes. Acta Neuropathol 99: 186-190, 2000

4. Koga Y, Akita Y, Takane N, Sato Y and Kato H: Heterogeneous presentation in A3243G mutation in the mitochondrial tRNA(Leu(UUR)) gene. Arch Dis Child 82: 407-411, 2000.

5. Iwanaga R, Koga Y, Aramaki S, Kato S and Kato H: Interand/or intra-organ distribution of mitochondrial C3303T or A3243G mutation in mitochondrial cytopathy. Acta Neuropathol 101: 179-184, 2001.

6. Olsson C, Zethelius B, Lagerström-Fermér M, Asplund J, Berne $C$ and Landegren U: Level of heteroplasmy for the mitochondrial mutation A3243G correlates with age at onset of diabetes and deafness. Hum Mutat 12: 52-58, 1998. 
7. Lu J, Wang D, Li R, Li W, Ji J, Zhao J, Ye W, Yang L, Qian Y, Zhu Y and Guan MX: Maternally transmitted diabetes mellitus associated with the mitochondrial tRNA(Leu(UUR)) A3243G mutation in a four-generation Han Chinese family. Biochem Biophys Res Commun 348: 115-119, 2006.

8. Rahman S, Poulton J, Marchington D and Suomalainen A: Decrease of $3243 \mathrm{~A} \rightarrow \mathrm{G}$ mtDNA mutation from blood in MELAS syndrome: a longitudinal study. Am J Hum Genet 68: 238-240, 2001.

9. Howell N, Ghosh SS, Fahy E and Bindoff LA: Longitudinal analysis of the segregation of mtDNA mutations in heteroplasmic individuals. J Neurol Sci 172: 1-6, 2000.
10. Olsson C, Johnsen E, Nilsson M, Wilander E, Syvänen AC and Lagerström-Fermér M: The level of the mitochondrial mutation A3243G decreases upon ageing in epithelial cells from individuals with diabetes and deafness. Eur J Hum Genet 9: 917-921, 2001.

11. 't Hart LM, Jansen JJ, Lemkes HH, De Knijff P and Maassen JA: Heteroplasmy levels of a mitochondrial gene mutation associated with diabetes mellitus decrease in leucocyte DNA upon aging. Hum Mutat 7: 193-197, 1996.

12. Shanske S, Pancrudo J, Kaufmann P, Engelstad K, Jhung S, Lu J, Naini A, DiMauro S and De Vivo DC: Varying loads of the mitochondrial DNA A3243G mutation in different tissues: implications for diagnosis. Am J Med Genet A 130A: 134-137, 2004. 